Gynaekologische Onkologie (AGO) has established a clinicopathological (Current Ovarian geRm cell and SEx cord stromal Tumour Treatment strategies, CORSETT) database for a better documentation and understanding of this rare disease. Here, we present the first descriptive analysis for patients with confirmed OGCT from the CORSETT database.

Methodology 20 German centres entered mixed retro- and prospective data of OGCT patients with histology specimens available treated between 2000 to 2014 into the CORSETT database. An independent CORSETT pathology reference panel checked the primary histological diagnosis. We conducted a descriptive analysis of the treatment strategies and created Kaplan-Meier curves and cox regression analyses for the survival analysis.

Results The reference pathology panel diagnosed 36 patients with dysgerminoma (FIGO stage $\mathrm{I}=77.8 \%$ ), 20 patients with mixed OGCT (FIGO stage $\mathrm{I}=66.7 \%$ ) and 21 with malignant teratoma (FIGO stage $\mathrm{I}=85 \%$ ). The median age of patients with dysgerminoma was 30.2 years (mixed OGCT: 35.6 and teratoma 36.4 years). 23 of dysgerminoma (63.8\%), six of mixed OGCT (31.6\%) and eight of teratoma (38.1\%) patients were treated with laparoscopy and the tumour ruptured intraoperatively in 21\% (dysgerminoma, mixed OGCT: 50\%, teratoma: $22 \%$ ) of the cases. 29 dysgerminoma (85.6\%), 15 mixed OGCT (78.9\%) and 17 teratoma (85\%) patients received fertility-sparing surgery. 20 of dysgerminoma (57\%), 14 of mixed OGCT (70\%) and 11 of teratoma (55\%) patients received adjuvant chemotherapy which decreased the likelihood of disease recurrence significantly in mixed OGCT patients to the highest degree (hazard ratio $=0.21,95 \%$ confidence interval $0.04-0.97)$. In total, two dysgerminoma (4.6\%), nine mixed OGCT (45\%) and three teratoma (14.3\%) patients experienced disease recurrence. The median progression-free and overall survival was not reached in the total cohort of OGCT patients.

Conclusion In this analysis, OGCT patients had an excellent prognosis despite non-negligible rates of intraoperative tumour spillages. Adjuvant chemotherapy appeared to prevent disease recurrence.

Disclosures

\section{PATIENT-REPORTED OUTCOMES (PROS) IN PATIENTS (PTS) RECEIVING NIRAPARIB IN THE PRIMA/ENGOT- OV26/GOG-3012 TRIAL}

\begin{abstract}
${ }^{1} J$ ohanna Mäenpää, ${ }^{2}$ Bhavana Pothuri, ${ }^{3}$ Sileny Han, ${ }^{4}$ Dana Chase, ${ }^{5}$ David Bender, ${ }^{6}$ Philippe Follana, ${ }^{7}$ Emeline Bacque, ${ }^{7} \mathrm{LI}$ Yong, ${ }^{8}$ Antonio González-Martin, ${ }^{8}$ Bradley J Monk. ${ }^{1}$ Tampere University; ' Gynecologic Oncology Group (Gog); Perlmutter Cancer Center, Nyu Langone Health; Department of Obstetrics/Gynecology; ${ }^{3}$ University Hospitals Leuven; Department of Obstetrics and Gynecology; ${ }^{4}$ Arizona Oncology (US Oncology Network), University of Arizona College of Medicine; ${ }^{5}$ University of lowa; Department of Obstetrics and Gynecology; ${ }^{6}$ Gineco; Centre Antoine Lacassagne; ${ }^{7}$ GlaxosmithklineGrupo Español de Investigación En Cáncer de Ovario (Geico); Clínica Universidad de Navarra; Medical Oncology Department; ${ }^{8}$ Arizona Oncology (US Oncology Network), University of Arizona College of Medicine
\end{abstract}

\subsection{6/ijgc-2020-ESGO.114}

Background Niraparib is a poly(ADP-ribose) polymerase (PARP) inhibitor that is approved for use in heavily pretreated pts and as maintenance treatment of pts with newly diagnosed or recurrent ovarian cancer following a response to platinumbased chemotherapy (CT). Here we report PROs in pts receiving niraparib and placebo (PBO) in the PRIMA/ENGOTOV26/GOG-3012 trial.

Methods This double-blind, PBO-controlled, phase 3 study randomised 733 pts with newly diagnosed advanced ovarian, primary peritoneal, or fallopian tube cancer with a complete or partial response (CR or PR) to first-line (1L) platinum-based CT. Pts received niraparib or $\mathrm{PBO}$ once daily for 36 months or until disease progression. The primary endpoint was progression-free survival (PFS) assessed by blinded independent central review. PROs, a secondary endpoint, were collected every 8 weeks for 56 weeks, then every 12 weeks thereafter while treatment was ongoing. Once a pt discontinued treatment, PRO evaluations were performed at the time of treatment discontinuation and then at $4,8,12$, and 24 weeks ( \pm 1 week for each time point) after the end of treatment, regardless of the status of subsequent treatment. The validated PRO instruments utilised were FOSI, EQ-5D-5L, EORTC-QLQ-C30, and EORTC-QLQ-OV28.

Results Compliance rates were high for all of the PRO instruments used in the study. PRO analysis of the EORTC-QLQC30 and EORTC-QLQ-OV28 did not indicate a difference in health-related quality of life scores of pts treated with niraparib vs placebo. Mean scores between niraparib and placebo arms were similar at each time point. Overall, the health utility index showed a slight improvement trend in pts who received niraparib vs placebo.

Conclusion Consistent with PRO results in the NOVA study, pts receiving niraparib in the PRIMA trial did not experience a decrease in quality of life compared with those receiving placebo.

Disclosures Sponsor: GlaxoSmithKline, Waltham, MA, USA

NCT number: NCT02655016

Encore statement: This data is presented on behalf of the original authors with their permission. Presented at the European Society for Medical Oncology (ESMO) Annual Meeting, September 19-21, 2020, Virtual.

Dr. Mäenpää reports Honoraria from Tesaro, AstraZeneca, Clovis, Roche, MSD and OrionPharma

Dr. Pothuri reports grants, personal fees and non-financial support from GSK; Advisory Board fees from AstraZeneca and Clovis Oncology.

Dr. Chase reports speakers' bureau fees from GSK.

Dr. González-Martín reports personal fees and non-financial support from AstraZeneca; Grant and personal fees from GSK, Clovis Oncology, Roche Holding AG, Merck \& Co., Inc., Genmab, INMUNOGEN, Pharma Mar, S.A., and Oncoinvent AS.

Dr. Monk reports consulting and advisory role at Merck, GSK, Roche/Genentech, AstraZeneca, Advaxiz, Cerulean Pharma, Amgen, Immunogen, NuCana BioMed, Clovis Oncology, Pfizer, Mateon Therapeutics, Precision Oncology, Perthera, Abbvie, Myriad Pharmaceuticals, Incyte, VBL Therapeutics, Takeda, Samumed, Oncomed, OncoSec, ChemoID, Geistlich Pharma, Eisai and Chemocare; Speakers' bureau at Roche/Genentech, AstraZeneca, Janssen, Clovis Oncology and GSK; Honoraria from Merck, GSK, Roche/Genentech, AstraZeneca, Advaxis, Immunogen, NuCana BioMed, Clovis Oncology, Pfizer, Mateon Therapeutics, Precision Oncology, Pethera, Abbvie, Myriad Pharmaceuticals, Incyte, Janssen, Amgen, Genmab, Samumed, Takeda, VBL Therapeutics, Puma Biotechnology, Immunomedics, Conjupro Biotherapeutics, Agenus, OncoQuest, ChemoID, Geistlich Pharma, Eisai and Chemocare; and Research funding from Novartis, Amgen, 
Genentech, Lilly, Janssen, Array BioPharma, GSK, Morphotek, Pfizer, Advaxis, AstraZeneca, Immunogen, Regeneron, and Nucana.

Drs. Han, Bender, and Follana have nothing to disclose.

Drs. Bacque and Li are employees of GlaxoSmithKline.

\section{NON-SURGICAL MANAGEMENT OF MALIGNANT BOWEL OBSTRUCTION IN ADVANCED OVARIAN CANCER PATIENTS - A SYSTEMATIC REVIEW AND META- ANALYSIS}

${ }^{1}$ Praveena Idaikkadar, ${ }^{2}$ Athina Georgiou, ${ }^{1}$ Simon Skene, ${ }^{1}$ Agnieszka Michael. ${ }^{1}$ University of Surrey; ${ }^{2}$ Kings College London

\subsection{6/ijgc-2020-ESG0.115}

Introduction/Background Ovarian cancer is the most lethal gynaecological malignancy and the 6 th most common cancer among women globally. The incidence of malignant bowel obstruction (MBO) in patients with advanced disease is up to $51 \%$. It presents a very distressing scenario for patients, their families and clinicians.

Management of $\mathrm{MBO}$ can be divided into surgical and medical management. Surgical management can involve direct resection, bypass surgery or stoma formation. Medical management includes endoscopic procedures, nasogastric tubes for decompression, bowel rest, parenteral feeding and symptom control such as chemotherapy, steroids, antisecretory drugs, analgesia and anti-emetics.

The rationale in choosing between surgical or medical management strategies is not well defined. High perioperative morbidity (up to 90\%) and mortality (up to 40\%) can make surgery a risky choice and there is increasing evidence that non-surgical management can significantly improve symptoms and quality of life.

The objective of this study was to evaluate the outcomes of patients with advanced ovarian cancer who undergo nonsurgical management of malignant bowel obstruction and conduct a meta-analysis to estimate median survival.

Methodology A literature search was carried out using the Pubmed, Embase and Medline online libraries up until November 2019. We also searched abstracts of scientific meetings, reference lists of included studies and contacted experts in the field. Relevant studies that met the inclusion criteria were independently selected by two of the co-authors and the data extracted and analysed separately.

Results In total 24 studies were found to be relevant for the systematic review and 9 met the eligibility criteria for the meta-analysis, a total of 2236 patients were included. Median survival for patients managed medically for bowel obstruction was 44 days (95\% CI 38-49 days, $\mathrm{I}^{2}=0 \%, \mathrm{P}$ $=0.128$ ).

Conclusion The quality of the included studies was relatively low, however the evidence shows that non-surgical management of bowel obstruction in advanced ovarian cancer patients results in a short survival period, but with controlled symptoms. Where quality of life is the main concern, this may be a feasible and effective strategy.

Disclosures Praveena Idaikkadar, Athina Georgiou and Simon Skene have no disclosures.

Agnieszka Michael has the following disclosures:

BMS, CLOVIS, ESAI, GSK, Ipsen, Novartis, Pfizer, Tesaro

\section{LEAP-005: EVALUATING THE SAFETY AND EFFICACY OF LENVATINIB AND PEMBROLIZUMAB IN PATIENTS PREVIOUSLY TREATED FOR OVARIAN CANCER, A MULTI-COHORT PHASE 2 STUDY}

${ }^{1}$ Antonio Gonzalez-Martin, ${ }^{2}$ Hyun Cheol Chung, ${ }^{3}$ Esma Saada-Bouzid, ${ }^{4}$ Eduardo Yanez, ${ }^{5}$ Hélène Senellart, ${ }^{6}$ Philippe A Cassier, ${ }^{7}$ Bristi Basu, ${ }^{8}$ Razi Ghori, ${ }^{9}$ Peter Kubiak, ${ }^{9}$ Alan Smith, ${ }^{8}$ Kevin Norwood, ${ }^{10}$ Zarnie Lwin. ${ }^{1}$ Clínica Universidad de Navarra; ${ }^{2}$ Yonsei Cancer Center, Yonsei University College of Medicine; ${ }^{3}$ Department of Medical Oncology, Centre de Lutte Contre Le Cancer Antoine Lacassagne; ${ }^{4}$ Oncology-Hematology Unit, Department of Internal Medicine, School of Medicine, Universidad de la Frontera; ${ }^{5}$ nstitut de Cancérologie de L'ouest, Centre René Gauducheau Ico; ${ }^{6}$ Department of Medical Oncology, Centre Léon Bérard; 'Department of Oncology, University of Cambridge; ${ }^{8}$ Merck and Co., Inc; ${ }^{9}$ Eisai Ltd., Hatfield, UK; ${ }^{10}$ Royal Brisbane and Women's Hospital, University of Queensland

\subsection{6/ijgc-2020-ESG0.116}

Introduction/Background Lenvatinib, an antiangiogenic multiple receptor tyrosine kinase inhibitor, plus pembrolizumab, a programmed death-1 immune checkpoint inhibitor, demonstrated promising clinical benefit in a previous phase $\mathrm{Ib} / \mathrm{II}$ trial across several cancer types (ClinicalTrials.gov, NCT02501096). We assessed clinical outcomes with lenvatinib plus pembrolizumab in patients with ovarian cancer in the ongoing, open-label, multicohort, phase II LEAP-005 study (ClinicalTrials.gov, NCT03797326).

\begin{tabular}{|c|c|c|c|}
\hline Studies & Est & imate $195 \%$ & $\because$ C.I.) \\
\hline Gadducci 1998 & 45.000 & $(30.300$, & $59.700)$ \\
\hline Bryan 2006 & 454.000 & (75.727, & $832.273)$ \\
\hline Mangili 1996 & 37.000 & $(23.045$, & $50.955)$ \\
\hline Matulonis 2005 & 89.000 & (14.913, & $163.087)$ \\
\hline Rath 2013 & 46.000 & (36.984, & 55.01 \\
\hline Pothuri 2004 & 56.000 & $(42.280$ & 69.72 \\
\hline Suidan 2017 & 36.000 & $(24.044$, & 47.95 \\
\hline Redman 1988 & 30.000 & $(-37.423$ & 97.42 \\
\hline DeEulis 2015 & 28.000 & $(-11 \cdot 395$ & 67.39 \\
\hline Overall $\left(I^{\wedge} 2=0 \%, P=0.128\right)$ & 43.965 & (38.652, & 49.27 \\
\hline
\end{tabular}

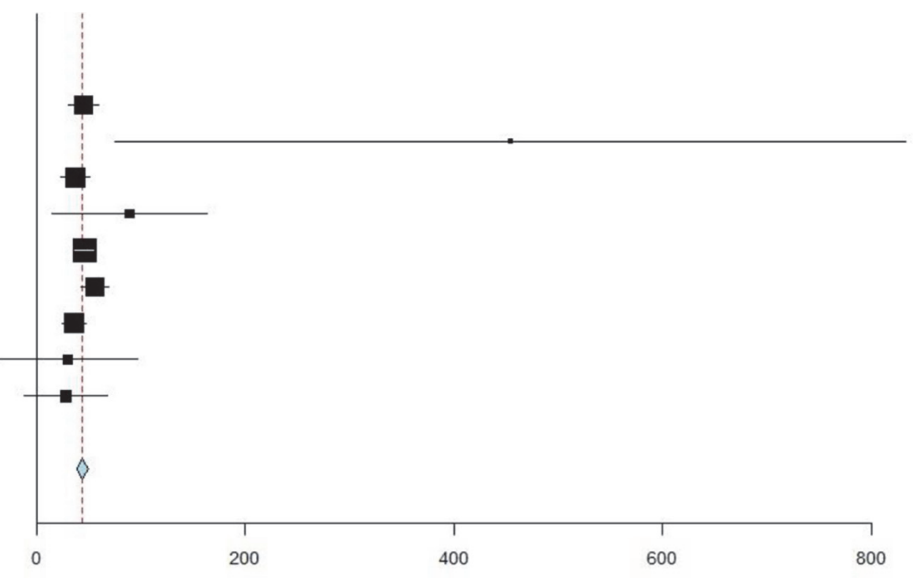

